\title{
Derivations Acting as Homomorphisms and as Anti-homomorphisms in $\sigma$-Lie Ideals of $\sigma$-Prime Gamma Rings
}

\author{
A. C. Paul ${ }^{1}$, S. Chakraborty ${ }^{2, *}$ \\ ${ }^{1}$ Department of Mathematics, Rajshahi University, Rajshahi-6205, Bangladesh \\ ${ }^{2}$ Department of Mathematics, Shahjalal University of Science and Technology, Sylhet-3114, Bangladesh
}

Copyright (C)2015 Horizon Research Publishing All rights reserved.

\begin{abstract}
Let $U$ be a non-zero $\sigma$-square closed Lie ideal of a 2-torsion free $\sigma$-prime $\Gamma$-ring $M$ satisfying the condition $a \alpha b \beta c=a \beta b \alpha c$ for all $a, b, c \in M$ and $\alpha, \beta \in \Gamma$, and let $d$ be a derivation of $M$ such that $d \sigma=\sigma d$. We prove here that (i) if $d$ acts as a homomorphism on $U$, then $d=0$ or $U \subseteq Z(M)$, where $Z(M)$ is the centre of $M$; and (ii) if $d$ acts as an anti-homomorphism on $U$, then $d=0$ or $U \subseteq Z(M)$.
\end{abstract}

Keywords $\sigma$-Prime Gamma Ring, Lie Ideal, Derivation, Involution

Mathematics Subject Classification 2010 16W10, 16W25, 16U80

\section{Introduction}

Suppose $M$ and $\Gamma$ are additive abelian groups. If there exists a mapping $(a, \alpha, b) \mapsto a \alpha b$ of $M \times \Gamma \times M \rightarrow M$ satisfying (a) $(a+b) \alpha c=a \alpha c+b \alpha c, a(\alpha+\beta) b=a \alpha b+a \beta b, a \alpha(b+c)=a \alpha b+a \alpha c$, and (b) $(a \alpha b) \beta c=a \alpha(b \beta c)$ for all $a, b, c \in M$ and $\alpha, \beta \in \Gamma$, then $M$ is said to be a $\Gamma$-ring in the sense of Barnes [3]. The set $Z(M)=\{a \in M: a \alpha m=m \alpha a$ for all $\alpha \in \Gamma$ and $m \in M\}$ is called the center of the $\Gamma$-ring $M$. In this article, $M$ will represent a $\Gamma$-ring with centre $Z(M)$.

Recall that $M$ is said to be 2-torsion free if $2 a=0$ with $a \in M$, then $a=0$. $M$ is called prime if, for any $a, b \in M$, $a \Gamma M \Gamma b=0$ implies $a=0$ or $b=0$. A mapping $\sigma: M \rightarrow M$ is called an involution if $\sigma^{2}(a)=a, \sigma(a+b)=\sigma(a)+\sigma(b)$ and $\sigma(a \alpha b)=\sigma(b) \alpha \sigma(a)$ for all $a, b \in M$ and $\alpha \in \Gamma$.

A $\Gamma$-ring $M$ equipped with an involution $\sigma$ is said to be a $\sigma$-prime $\Gamma$-ring if for all $a, b \in M, a \Gamma M \Gamma b=0=a \Gamma M \Gamma \sigma(b)$ implies $a=0$ or $b=0$. It is noted that every prime $\Gamma$-ring having an involution $\sigma$ is $\sigma$-prime, but the converse is in general not true. Let $S_{a \sigma}(M)=\{a \in M: \sigma(a)= \pm a\}$, which represents the set of symmetric and skew-symmetric elements of $M$.

For any $a, b \in M$ and $\alpha \in \Gamma$, the symbol $[a, b]_{\alpha}$ stands for the commutator $a \alpha b-b \alpha a$. The basic commutator identities are

$$
\begin{gathered}
{[a \beta b, c]_{\alpha}=a \beta[b, c]_{\alpha}+a[\beta, \alpha]_{c} b+[a, c]_{\alpha} \beta b \text { and }} \\
{[a, b \beta c]_{\alpha}=b \beta[a, c]_{\alpha}+b[\beta, \alpha]_{a} c+[a, b]_{\alpha} \beta c,}
\end{gathered}
$$


where $[\alpha, \beta]_{a}=\alpha a \beta-\beta a \alpha$, for all $a, b, c \in M$ and $\alpha, \beta \in \Gamma$. Throughout the article, we shall consider the condition

$$
\text { (*) } a \alpha b \beta c=a \beta b \alpha c
$$

for all $a, b, c \in M$ and $\alpha, \beta \in \Gamma$. Using this condition (*), the above identities reduce to

$$
[a \beta b, c]_{\alpha}=a \beta[b, c]_{\alpha}+[a, c]_{\alpha} \beta b \text { and }[a, b \beta c]_{\alpha}=b \beta[a, c]_{\alpha}+[a, b]_{\alpha} \beta c,
$$

which are extensively used in our results.

An additive subgroup $U$ of $M$ is called a left (or, right) ideal of $M$ if $M \Gamma U \subset U$ (or, $U \Gamma M \subset U$ ), whereas $\mathrm{U}$ is called a (two-sided) ideal of $M$ if $U$ is a left as well as a right ideal of $M$.

An additive subgroup $U$ of $M$ is called a Lie ideal if $[U, M]_{\Gamma} \subset U$. If $U$ is a Lie ideal of $M$, then $U$ is called a $\sigma$-Lie ideal if $\sigma(U)=U$, and $U$ is called a square closed Lie ideal if $u \alpha u \in U$ for all $u \in U$ and $\alpha \in \Gamma$. A Lie ideal $U$ of $M$ is said to be a $\sigma$-square closed Lie ideal if it is square closed and $\sigma(U)=U$.

An additive mapping $d: M \rightarrow M$ is called a derivation if $d(a \alpha b)=d(a) \alpha b+a \alpha d(b)$ for all $a, b \in M$ and $\alpha \in \Gamma$. An additive mapping $\phi: M \rightarrow M$ is said to be a homomorphism if $\phi(a \alpha b)=\phi(a) \alpha \phi(b)$ for all $a, b \in M$ and $\alpha \in \Gamma$. And, an additive mapping $\psi: M \rightarrow M$ is called an anti-homomorphism if $\psi(a \alpha b)=\psi(b) \alpha \psi(a)$ for all $a, b \in M$ and $\alpha \in \Gamma$.

A derivation $d$ of $M$ is said to act as a homomorphism [resp. as an anti-homomorphism] on a subset $S$ of $M$ if $d(a \alpha b)=$ $d(a) \alpha d(b)$ [resp. $d(a \alpha b)=d(b) \alpha d(a)]$ for all $a, b \in S$ and $\alpha \in \Gamma$.

In [4], Bell and Kappe proved that if $d$ is a derivation of a semiprime ring $R$ which is either an endomorphism or an anti-endomorphism on $R$, then $d=0$; whereas, the behavior of $d$ is somewhat restricted in case of prime rings in the way that if $d$ is a derivation of a prime ring $R$ acting as a homomorphism or an anti-homomorphism on a non-zero right ideal $U$ of $R$, then $d=0$. Asma et. al. [1] extended this result of prime rings on square closed Lie ideals. Afterwards, the said result was extended to $\sigma$-prime rings by Oukhtite et. al. in [11].

In $\Gamma$-rings, Dey and Paul [9] proved that if $D$ is a generalized derivation of a prime $\Gamma$-ring $M$ with an associated derivation $d$ of $M$ which acts as a homomorphism and an anti-homomorphism on a non-zero ideal $I$ of $M$, then $d=0$ or $M$ is commutative. Afterwards, Chakraborty and Paul [6] worked on $k$-derivation of a semiprime $\Gamma$-ring in the sense of Nobusawa [10] and proved that $d=0$ where $d$ is a $k$-derivation acting as a $k$-endomorphism and as anti-k-endomorphism.

In this article, the above mentioned results following [11] in classical rings are extended to those in gamma rings with derivation acting as a homomorphism and as an anti-homomorphism on $\sigma$-prime $\Gamma$-rings. Our objective is to prove that

(i) if $d$ is a derivation of a 2-torsion free $\sigma$-prime $\Gamma$-ring $M$ such that $d \sigma=\sigma d$ and if $d$ acts as a homomorphism on a non-zero $\sigma$-square closed Lie ideal $U$ of $M$, then $d=0$ or $U \subseteq Z(M)$; and

(ii) if $d$ is a derivation of a 2-torsion free $\sigma$-prime $\Gamma$-ring $M$ satisfying the condition (*) such that $d \sigma=\sigma d$ and if $d$ acts as an anti-homomorphism on a non-zero $\sigma$-square closed Lie ideal $U$ of $M$, then $d=0$ or $U \subseteq Z(M)$.

\section{Derivation acting as a homomorphism and as an anti-homomorphism of $\sigma$ - prime $\Gamma$-rings}

We start this section with an example which ensures the existence of an involution in a $\Gamma$-ring. We also give an example of a $\sigma$-prime $\Gamma$-ring which is not a prime $\Gamma$-ring along with an example of a Lie ideal in a $\Gamma$-ring.

Example 2.1 Let $M$ be a $\Gamma$-ring. Define $M_{1}=\{(a, b): a, b \in M\}$ and $\Gamma_{1}=\{(\alpha, \alpha): \alpha \in \Gamma\}$. Addition and multiplication on $M_{1}$ are defined as: $(a, b)+(c, d)=(a+c, b+d)$ and $(a, b)(\alpha, \alpha)(c, d)=(a \alpha c, d \alpha b)$. Under these addition and multiplication, $M_{1}$ is a $\Gamma_{1}$-ring. Define a mapping $\sigma: M_{1} \rightarrow M_{1}$ by $\sigma((a, b))=(b, a)$. Then $\sigma$ is an involution on $M_{1}$ ([13], Example 3.2).

In this case, if $M$ is a prime $\Gamma$-ring, then we claim that $M_{1}$ equipped with the above involution $\sigma$ is a $\sigma$-prime $\Gamma_{1}$-ring.

Because, for any $(a, b),(c, d),(x, y) \in M_{1}$ and $(\alpha, \alpha),(\beta, \beta) \in \Gamma_{1}$, we assume that $(a, b)(\alpha, \alpha)(x, y)(\beta, \beta)(c, d)=0=$ $(a, b)(\alpha, \alpha)(x, y)(\beta, \beta)(d, c)$ [since $\sigma((c, d))=(d, c)$ ]. After calculation, it gives: $a \alpha x \beta c=0, d \beta y \alpha b=0, a \alpha x \beta d=0$ and $c \beta y \alpha b=0$ for all $x, y \in M$ and $\alpha, \beta \in \Gamma$; and therefore, $a \Gamma M \Gamma c=0, d \Gamma M \Gamma b=0, a \Gamma M \Gamma d=0$ and $c \Gamma M \Gamma b=0$. In view 
of the primeness of $M$, these yield: $a=0$ or $c=0 ; d=0$ or $b=0 ; a=0$ or $d=0$ and $c=0$ or $b=0$. In all the cases, we obtain $(a, b)=0$ or $(c, d)=0$, which establishes our claim.

But, $M_{1}$ is not a prime $\Gamma$-ring. For, $(a, 0)(\alpha, \alpha)(x, y)(\beta, \beta)(0, b)=(0,0)$ but $(a, 0)$ or $(0, b)$ are not zero.

Example 2.2 Let $R$ be a commutative ring of characteristic 2 having unity element 1 . Consider $M=M_{1,2}(R)$ and $\Gamma=$ $\left\{\left(\begin{array}{c}n .1 \\ n .1\end{array}\right): n \in Z, 2 \nmid n\right\}$. Then $M$ is a $\Gamma$-ring. Suppose $N=\{(x, x): x \in R\} \subseteq M$. Then for each $(x, x) \in N$, $(a, b) \in M$ and $\left(\begin{array}{c}n \\ n\end{array}\right) \in \Gamma$, we have

$$
\begin{gathered}
(x, x)\left(\begin{array}{c}
n \\
n
\end{array}\right)(a, b)-(a, b)\left(\begin{array}{c}
n \\
n
\end{array}\right)(x, x) \\
=(x n a-b n x, x n b-a n x) \\
=(x n a-2 b n x+b n x, b n x-2 a n x+x n a) \\
=(x n a+b n x, b n x+x n a) \in N .
\end{gathered}
$$

Therefore, $N$ is a Lie ideal of $M$.

If $U$ is a square closed Lie ideal (i.e. for all $u \in U$ and $\alpha \in \Gamma$ ), then for each $v \in U, u \alpha v+v \alpha u=(u+v) \alpha(u+v)-$ $u \alpha u-v \alpha v$. Therefore, $u \alpha v+v \alpha u \in U$. On the other hand, $u \alpha v-v \alpha u \in U$ for all $u, v \in U$ and $\alpha \in \Gamma$. Hence, $2 u \alpha v \in U$ for all $u, v \in U$ and $\alpha \in \Gamma$. We need to use this result frequently.

We proceed with the following lemmas.

Lemma 2.1 ([8], Lemma 3.1) Let $U \neq 0$ be a $\sigma$-ideal of a 2-torsion free $\sigma$-prime $\Gamma$-ring $M$ satisfying the condition (*). If $[U, U]_{\Gamma}=0$, then $U \subseteq Z(M)$.

Lemma 2.2 ([7], Lemma 2.2) Let $U \nsubseteq Z(M)$ be a $\sigma$-ideal of a 2-torsion free $\sigma$-prime $\Gamma$-ring $M$ satisfying the condition (*) and $a, b \in M$ such that $a \alpha U \beta b=a \alpha U \beta \sigma(b)=0$ for all $\alpha, \beta \in \Gamma$. Then $a=0$ or $b=0$.

Lemma 2.3 Let $U \neq 0$ be a $\sigma$-ideal of a 2-torsion free $\sigma$-prime $\Gamma$-ring $M$ satisfying the condition $(*)$ and $d$ a derivation of $M$ such that $d \sigma=\sigma d$ and $d(U)=0$. Then $d=0$ or $U \subseteq Z(M)$.

Proof. For all $u \in U, m \in M$ and $\alpha \in \Gamma$, we have $[u, m]_{\alpha} \in U$. So, we get $0=d\left([u, m]_{\alpha}\right)=[d(u), m]_{\alpha}+$ $[u, d(m)]_{\alpha}=[u, d(m)]_{\alpha}$. That is, for all $u \in U, m \in M$ and $\alpha \in \Gamma$,

$$
[u, d(m)]_{\alpha}=0
$$

Putting $m \beta t$ for $m$ in (1), where $t \in M$ and $\beta \in \Gamma$, we have

$$
\begin{gathered}
0=[u, d(m \beta t)]_{\alpha}=[u, d(m) \beta t+m \beta d(t)]_{\alpha} \\
=d(m) \beta[u, t]_{\alpha}+[u, d(m)]_{\alpha} \beta t+[u, m]_{\alpha} \beta d(t)+m \beta[u, d(t)]_{\alpha} \\
=d(m) \beta[u, t]_{\alpha}+[u, m]_{\alpha} \beta d(t), \text { by using (1). }
\end{gathered}
$$

Thus, for all $u \in U, m, t \in M$ and $\alpha, \beta \in \Gamma$, we have

$$
d(m) \beta[u, t]_{\alpha}+[u, m]_{\alpha} \beta d(t)=0 .
$$

Taking $t=m$ in (2), we find that

$$
d(m) \beta[u, m]_{\alpha}+[u, m]_{\alpha} \beta d(m)=0
$$

Since $d(m) \beta[u, m]_{\alpha}=[u, m]_{\alpha} \beta d(m)$ [by (1)], therefore, we have

$$
2 d(m) \beta[u, m]_{\alpha}=0
$$

By the 2-torsion freeness of $M$, for all $u \in U, m \in M$ and $\alpha, \beta \in \Gamma$, we obtain

$$
d(m) \beta[u, m]_{\alpha}=0 .
$$

Replacing $u$ by $2 u \gamma v$ in (3), with $v \in U$ and $\gamma \in \Gamma$, we have

$$
0=d(m) \beta[2 u \gamma v, m]_{\alpha}
$$




$$
\begin{gathered}
=2 d(m) \beta u \gamma[v, m]_{\alpha}+2 d(m) \beta[u, m]_{\alpha} \gamma v \\
=2 d(m) \beta u \gamma[v, m]_{\alpha}, \text { by using (3). }
\end{gathered}
$$

Since $M$ is 2-torsion free, for all $v \in U, m \in M$ and $\alpha, \beta, \gamma \in \Gamma$, we obtain

$$
d(m) \beta U \gamma[v, m]_{\alpha}=0 .
$$

Let $m \in S_{a \sigma}(M)$. Then the fact that $\sigma(U)=U$ leads to

$$
d(m) \beta U \gamma[v, m]_{\alpha}=0=d(m) \beta U \gamma \sigma\left([v, m]_{\alpha}\right) .
$$

In view of Lemma 2.2, it gives that $d(m)=0$ or $[v, m]_{\alpha}=0$ for all $v \in U$. As $m+\sigma(m) \in S_{a \sigma}(M)$, then $d(m+\sigma(m))=0$ or $[U, m+\sigma(m)]_{\alpha}=0$. If $[U, m+\sigma(m)]_{\alpha}=0$, then $[U, m]_{\alpha}=-[U, \sigma(m)]_{\alpha}$. If $[U, m-\sigma(m)]_{\alpha}=0$, then $[U, m]_{\alpha}=$ $[U, \sigma(m)]_{\alpha}$. Adding these two relations, we obtain that $2[U, m]_{\alpha}=0$, and hence $[U, m]_{\alpha}=0$ for all $m \in M$ and $\alpha \in \Gamma$, by 2-torsion freeness of $M$.

Now we assume that $d(m+\sigma(m))=0$. It gives $d(m)+\sigma d(m))=0$ (since $d \sigma=\sigma d$ ), and we obtain $d(m) \in S_{a \sigma}(M)$. Applying this in (4), we conclude that $d(m)=0$ or $[U, m]_{\alpha}=0$. If $d(m-\sigma(m))=0$, then $d(m) \in S_{a \sigma}(M)$, and once again by using (4), we obtain that $d(m)=0$ or $[U, m]_{\alpha}=0$.

Let $A=\{m \in M: d(m)=0\}$ and $B=\left\{m \in M:[U, m]_{\alpha}=0\right\}$. Then $A$ and $B$ are two additive subgroups of $M$ such that $A \subset M$ and $B \subset M$. We also have $A \cup B=M$. But, a group cannot be a union of two of its proper subgroups, and thus $M=A$ or $M=B$. If $M=A$, then $d(m)=0$ for all $m \in M$, i.e. $d=0$. If $M=B$, then $U \subseteq Z(M)$. Consequently, we have $d=0$ or $U \subseteq Z(M)$.

Now we have the position to prove our main results.

Theorem 2.1 Let $U \neq 0$ be a $\sigma$-square closed Lie ideal of a 2-torsion free $\sigma$-prime $\Gamma$-ring $M$ satisfying the condition (*) and $d$ a derivation of $M$ which acts as a homomorphism on $U$. If $d \sigma=\sigma d$, then $d=0$ or $U \subseteq Z(M)$.

Proof. Let us suppose that $d(a \alpha b)=d(a) \alpha d(b)$ for all $a, b \in U$ and $\alpha \in \Gamma$. Assume that $a, b, c \in U$ and $\alpha, \beta \in \Gamma$. As $4 a \alpha b \beta c=2(2 a \alpha b) \beta c$, it follows that $4 a \alpha b \beta c \in U$. Since $M$ is 2-torsion free, we obtain

$$
d(a \alpha b \beta c)=d(a \alpha b) \beta c+a \alpha b \beta d(c)=d(a) \alpha d(b) \beta c+a \alpha b \beta d(c) .
$$

On the other hand,

$$
d(a \alpha b \beta c)=d(a) \alpha d(b \beta c)=d(a) \alpha d(b) \beta c+d(a) \alpha b \beta d(c) .
$$

Comparing (6) and (7), we obtain $(d(a)-a) \alpha b \beta d(c)=0$ for all $a, b, c \in U$ and $\alpha, \beta \in \Gamma$. Therefore, for all $a, c \in U$ and $\alpha, \beta \in \Gamma$, we get

$$
(d(a)-a) \alpha U \beta d(c)=0 .
$$

As $d \sigma=\sigma d$ and $\sigma(U)=U$, we conclude that $d(c)=0$ for all $c \in U$ or, $d(a)=a$ for all $a \in U$. If $d(c)=0$ for all $c \in U$, then in view of Lemma 2.3, we conclude that $d=0$ or $U \subseteq Z(M)$. Now consider $d(a)=a$ for all $a \in U$. Let $m \in M$, $u \in U$ and $\alpha \in \Gamma$. Using $d(u)=u$ and $d\left([u, m]_{\alpha}\right)=[u, m]_{\alpha}$, we have seen that $[u, d(m)]_{\alpha}=0$ for all $u \in U, m \in M$ and $\alpha \in \Gamma$. By the similar argument as in the proof of Lemma 2.3, we are forced to conclude that $d=0$ or $U \subseteq Z(M)$.

Theorem 2.2 Let $M$ be a 2-torsion free $\sigma$-prime $\Gamma$-ring satisfying the condition (*), and let $U \neq 0$ be a $\sigma$-square closed Lie ideal of $M$. Let $d$ be a derivation of $M$ which acts as an anti-homomorphism on $U$. If $d \sigma=\sigma d$, then $d=0$ or $U \subseteq Z(M)$.

Proof. Suppose that $d$ acts as an anti-homomorphism on $U$. For all $a, b \in U$ and $\alpha \in \Gamma$, we then get

$$
d(a \alpha b)=d(a) \alpha b+a \alpha d(b)=d(b) \alpha d(a) .
$$

Substituting $2 a \beta b$ for $a$ in (9) with $\beta \in \Gamma$, and using the 2-torsion freeness of $M$, we get

$$
d(a \beta b) \alpha b+a \beta b \alpha d(b)=d(b) \alpha d(a \beta b)
$$




$$
\Longrightarrow d(b) \beta d(a) \alpha b+a \beta b \alpha d(b)=d(b) \alpha d(a) \beta b+d(b) \alpha a \beta d(b) .
$$

By using (*), for all $a, b \in U$ and $\alpha, \beta \in \Gamma$, we then obtain

$$
a \alpha b \beta d(b)=d(b) \beta a \alpha d(b)
$$

Replacing $a$ by $2 c \gamma a$ in (10), where $c \in U$ and $\gamma \in \Gamma$, and using the 2-torsion freeness of $M$, we find that

$$
c \gamma a \alpha b \beta d(b)=d(b) \beta c \gamma a \alpha d(b)
$$

for all $a, b, c \in U$ and $\alpha, \beta, \gamma \in \Gamma$. But, from (10), we get

Comparing this with (11), we obtain

$$
c \gamma a \alpha b \beta d(b)=c \gamma d(b) \beta a \alpha d(b) .
$$

$$
c \gamma d(b) \beta a \alpha d(b)=d(b) \beta c \gamma a \alpha d(b) .
$$

By using (*), we find that

$$
[c, d(b)]_{\gamma} \beta a \alpha d(b)=0,
$$

and hence, for all $b, c \in U$ and $\alpha, \beta, \gamma \in \Gamma$, we get

$$
[c, d(b)]_{\gamma} \beta U \alpha d(b)=0
$$

For $b \in U \cap S_{a \sigma}(M)$, as $\sigma(U)=U$, we have

$$
[c, d(b)]_{\gamma} \beta U \alpha d(b)=0=[c, d(b)]_{\gamma} \beta U \alpha \sigma(d(b)) .
$$

In view of Lemma 2.2, we obtain $[c, d(b)]_{\gamma}=0$ or $d(b)=0$ for all $c \in U$. Since $d \sigma=\sigma d$ and $\sigma(U)=U$, using the fact that $c+\sigma(c), c-\sigma(c) \in U \cap S_{a \sigma}(M)$, by virtue of (12), we clearly find that $d(b)=0$ or $[U, d(b)]_{\gamma}=0$ for all $b \in U$ and $\gamma \in \Gamma$.

Set $F=\{b \in U: d(b)=0\}$ and $G=\left\{b \in U:[U, d(b)]_{\gamma}=0\right\}$. Clearly, $F$ and $G$ are additive subgroups of $U$ such that $U=F \cup G$, and hence $U=F$ or $U=G$. If $U=F$, then $d(U)=0$, and by virtue of Lemma 2.3 , we find that $d=0$ or $U \subseteq Z(M)$. Now assume that $U=G$. Then, for all $u \in U$, we obtain

$$
[u, d(b)]_{\gamma}=0
$$

Putting $b \alpha b$ for $b$ in (13), we get

$$
\begin{gathered}
0=[u, d(b \alpha b)]_{\gamma}=[u, d(b) \alpha b+b \alpha d(b)]_{\gamma} \\
=d(b) \alpha[u, b]_{\gamma}+[u, d(b)]_{\gamma} \alpha b+b \alpha[u, d(b)]_{\gamma}+[u, b]_{\gamma} \alpha d(b) .
\end{gathered}
$$

By using (13) in the above relation, we obtain

$$
d(b) \alpha[u, b]_{\gamma}+[u, b]_{\gamma} \alpha d(b)=0 .
$$

From (13), we get $u \gamma d(b)=d(b) \gamma u$, which forces to $d(b) \alpha[u, b]_{\gamma}=[u, b]_{\gamma} \alpha d(b)$, since $[u, b]_{\gamma} \in U$. Using this in (14), by the 2-torsion freeness of $M$, for all $u, b \in U$ and $\alpha, \gamma \in \Gamma$, we find

$$
d(b) \alpha[u, b]_{\gamma}=0
$$

Putting $2 u \beta v$ in place of $u$, where $v \in U$ and $\beta \in \Gamma$, and using (15) and 2-torsion freeness of $M$, we get $d(b) \alpha u \beta[v, b]_{\gamma}=0$ so that $d(b) \alpha U \beta[v, b]_{\gamma}=0$ for all $b, v \in U$ and $\alpha, \beta, \gamma \in \Gamma$.

Let $b \in U \cap S_{a \sigma}(M)$, as $\sigma(U)=U$, we obtain $d(b)=0$ or $[v, b]_{\gamma}=0$ for all $v \in U$. Thus, by the similar reasoning as above, one can easily see that $d(b)=0$ or $[U, b]_{\gamma}=0$ for all $b \in U$ and $\gamma \in \Gamma$. Consequently, $U$ is a union of two additive subgroups $G$ and $H$, where $G=\{b \in U: d(b)=0\}$ and $H=\left\{b \in U:[U, b]_{\gamma}=0\right\}$, and thus $U=G$ or $U=H$. If $U=G$, then $d(U)=0$, and Lemma 2.3 forces that $d=0$ or $U \subseteq Z(M)$. On the other hand, if $U=H$, then $[c, t]_{\gamma}=0$ for all $c, t \in U$ and $\gamma \in \Gamma$. In this case, we have $[U, U]_{\Gamma}=0$, and so $U \subseteq Z(M)$, by Lemma 2.1. Thus, in all the cases we have seen that $d=0$ or $U \subseteq Z(M)$, completing the proof. 


\section{Acknowledgements}

We are thankful to the reviewers for their useful suggestions and valuable comments to improve this article significantly.

\section{REFERENCES}

[1] A. Asma, N. Rehman and A. Shakir, On Lie ideals with derivations as homomorphisms and anti-homomorphisms, Acta Math. Hungar., 101(1-2) (2003), 79-82.

[2] A. Ali and D. Kumar, Derivation which acts as a homomorphism or as an anti-homomorphism in a prime ring, Internat. Math. Forum, 2(23) (2007), 1105-1110.

[3] W. E. Barnes, On the Г-Rings of Nobusawa, Pacific J. Math., 18 (1966), 411-422.

[4] H. E. Bell and L. C. Kappe, Rings in which derivations satisfy certain algebraic conditions, Acta Math. Hungar., 53(3-4) (1989), $339-346$.

[5] S. Chakraborty and A. C. Paul, On Jordan $k$-isomorphisms of certain $\Gamma_{N}$-rings, Indian J. Math., 53(1) (2011), 99-111.

[6] S. Chakraborty and A. C. Paul, The $k$-derivation acting as a $k$-endomorphism and as an anti-k-endomorphism on semiprime Nobusawa gamma ring, GANIT J. Bangladesh Math. Soc., 33 (2013), 93-101.

[7] K. K. Dey, A. C. Paul and B. Davvaz, On commutativity of $\sigma$-prime $\Gamma$-rings, Kyungpook Math. J. (to appear).

[8] K. K. Dey, A. C. Paul and B. Davvaz, On centralizing automorphisms and Jordan left derivations on $\sigma$-prime gamma rings, Hacettepe J. Math. Stat. (to appear).

[9] K. K. Dey and A. C. Paul, Generalized derivations acting as homomorphisms and anti-homomorphisms of gamma rings, J. Sci. Res., 4(1) (2012), 33-37.

[10] N. Nobusawa, On a Generalization of the Ring Theory, Osaka J. Math., 1 (1964), 81-89.

[11] L. Oukhtite, S. Salhi and L. Taoufiq, $\sigma$-Lie ideals with derivations as homomorphisms and anti-homomorphisms, Internat. J. Algebra, 1(5) (2007), 235-239.

[12] L. Oukhtite, S. Salhi and L. Taoufiq, Derivations and commutativity of $\sigma$-prime rings, Glasnik Mathematicki, 41(1) (2006), 57-64.

[13] A. C. Paul and M. S. Uddin, Lie Structure in simple gamma rings with involutions, J. Calcutta Math. Soc., 9(2) (2013), 87-100. 